\title{
Analysis of the factors that restrict the career women's participation in sports
}

\author{
Jin Xiaoli \\ Xingtai Medical College, Hebei, China 054000 \\ fsyyjcs@126.com
}

Keywords: career women; sports; restrict

\begin{abstract}
This paper aims to analyze the factors that restrict the career women's participation in sports, including sports values, sports skill, the impact of leisure time, income, family support, social sports atmosphere, construction of community sports organizations and community sports facilities, etc.
\end{abstract}

\section{Introduction}

Development of anything comes from a variety of factors. Like men, career women need to face all kinds of challenges and difficulties as an important force in the labor market of our country. Women's participation in sports is a sign of women's liberation[1]. Not the same as men, career women need to play a more important role in the family because of the impact of China's traditional culture, which also face various problems because of the difference from men in physiology and psychology. For this reason, the female needs more humanistic care in the social position, especially physical exercises directly related to women's physical and mental health. The restricting factors of career women's participation in sports are as follows.

\section{Sports values}

The conception of sports value is human values embodied in sports; is one of the important contents of people's sports awareness and understanding; is the guidance, control and evaluation of sports behavior and effectiveness of the core concept. Proper sports value is an important factor to encourage people to participate in the mass sports activities. Career women have high recognition value of sports, such as physical fitness, bodybuilding plastic, resistance improvement and other aspects of the sports value, while the degree of social recognition given to the value of sports is relatively low, such as meeting the personal interests, harmonious family relations, enhanced aesthetic tastes and so on. The value of sports identity will directly affect the sports participation enthusiasm and interest, and also affect the formation of occupation women's physical exercise habits and lifelong sports consciousness.

\section{Sports skill}

Sports skills mastered in physical exercise can help participants play physical potential better and show a combination of strength and beauty, so as to meet the psychological needs and improve the physiological quality. The vast majority of professional women received education above the high school system, including physical education. They should master certain spots skill during the school. However, long-term neglect of exercise or incorrect personal sports concept will cause that these movement skills are difficult to play their roles in today's physical exercise. When the professional women are doing exercise, the lack of sports skills will make them low fitness effect and low efficiency, and also make fitness activities lack beauty and charm, which directly affect the confidence and perseverance of fitness. Therefore, this factor is very important for the influence on professional women's participation in sports. 


\section{Impact of leisure time}

For professional women, the value of sport can not be overlooked, especially when they are seeking for a peaceful and relaxing space in the cracks of today's accelerating pace of work and life, sport is a good choice. But career women do not have enough leisure time, as they bear more social and family work so that they neglect their spiritual enjoyment. Another reason is the lack of publicity and organization of work, which will lead to the proportion of working women's leisure time used for physical exercise is too little.

\section{Income}

As one of the important lifestyle for relaxation, sports need strong financial support. From buying sports clothing and equipment to becoming a fitness club membership in the current fashion life, are inseparable from economic expenditure. Therefore, among the factors of influencing career women's physical exercise, the role of economic income is very obvious. The proportion of educated women participating in sports consumption is not high and the factors affecting educated women's sports consumption are personal income level, physical ability, quality of sports products, sports culture and environmental factors [2].

\section{Family support}

Development of women's sports can promote family harmony and maintain social stability [3]. Occupation female is an important composition of the family, shouldering the important responsibility for family. Married women, especially working women with children, have more difficult families work. Facing fast-paced work at work time, needing to clean up home chores and educating their children, therefore, women are very difficult to have plenty of time and efforts to carry out their own leisure and recreation, improve the level of spiritual life. Bottleneck to solve this problem is undoubtedly to reduce the liability in the family, so that other family members share the responsibility for household and education of children, providing selfless support from the family for professional women's sports fitness behavior.

\section{Social sports atmosphere}

With the progress of times, the social status of Chinese women has undergone enormous changes, which are also reflected in the participation of women in physical exercise [4]. "Women's enthusiasm for participating in sports is not directly dependent on the concept of women's culture changes, but ultimately depends on social productivity and economic development level” [5]. With social progress and economic development, the material life of our residents has increased dramatically. People began to pursue higher spiritual enjoyment, so how to have a healthier life has become one of the focus of the current national concern. Sports atmosphere of the whole society will make them quickly recognize, accept and dedicate themselves to this healthy lifestyle. Meanwhile, some policies and regulations concerning the protection of women's health rights have also introduced which provide a good guarantee for the smooth progress of women's sports. Sports public opinion of various media played an important role in creating a social atmosphere in sports. People will have a better understanding and preference of a sports project by the attraction of sports stars. As educated Women in the new era, professional women play a remarkable role in feeling the charm and value of sports.

\section{Construction of community sports organizations}

All kinds of sports organizations in our country play an important role in continuously promoting the national fitness project. In urban communities, these health organizations can gather different numbers of people to do sports exercises and they made an indelible contribution to the 
successfully carrying out the work of community sports. Professional women not only can achieve the core values of sport, but also can fully embody the value of sports that society gives by participating in these organized activities in their spare time, such as improving relationship, meeting personal preferences, building up self-confidence, harmonious family relationships, enhancing aesthetics.

\section{The construction of community sports facilities}

Physical fitness activities can not do without the facilities support. Currently, the community overall fitness path, some simple equipment has provided a better workout condition for residents, but generally speaking, the fitness equipment can not meet the needs for sports created by professional women. It's difficult to provide all the fitness equipment which career women are more favorite. Health club has complete facilities, but career women will not choose it because they need to pay much money. So career women often like to go to parks, community open space and public sports venues. But there are no specialized fitness space and facilities in the community open area and nearby park square, likewise, facilities were overwhelmed, seriously damaged in the public sports venues. The material factor significantly restricts the career women's participation in sports activities. Therefore, the relevant departments should increase investment and overall improve the exercise environment.

\section{Summary}

As women in the new era, career women's sports participation behavior directly affects the development of women's sports in China, also influence the smooth implementation of the national fitness program. So strengthening the research on female sports to the society, family, individual has important theoretical value and practical significance.

\section{References}

[1] Zhang qing wu ,Yin weili ,Zhang deli, etc.: The current situation and thinks of female physical research in China... based on CNKI data analysis, Physicals and Science [J]. 2011(2): 32

[2] Yu Yun xia The research of the consume of female physicals in Shang hai [J]. Market moderation 2009(9): 183-184.

[3] Xu gui lan ,liu xinmin The female physical research under the condition of harmonious society [J]. Hebei physical college paper 2010, 24(2):5-7

[4] Huang jun, Liu lianfa, The relevant analysis of the current situation and its factors of urban female physical practices-based on Beijing city [J]. Physicals and science 2010, 24(2):5-7

[5] Done jin xia, The research of female physicals in China [J]. Physicals and science 2007(1): 2 